\title{
Population Health Management during Student Pharmacist Introductory Experiential Education to Expand Clinical Pharmacist Impact
}

\author{
Macy McConnell, PharmD ${ }^{1,2}$; Daniel J. Mobley, PharmD ${ }^{1,2,4}$; Alex Gidal, PharmD ${ }^{1,2,5}$; Michael W. Nagy, PharmD ${ }^{1,3,5}$ \\ ${ }^{1}$ William S. Middleton Memorial Veteran Affairs Hospital; ${ }^{2}$ University of Wisconsin-Madison School of Pharmacy; \\ ${ }^{3}$ Medical College of Wisconsin School of Pharmacy; ${ }^{4}$ VA Puget Sound Health Care System; ${ }^{5}$ Clement J. Zablocki Veterans Affairs \\ Medical Center
}

\begin{abstract}
Objective: To evaluate the impact of incorporating pre-advanced pharmacy practice experience (pre-APPE) student pharmacists into three different population health management (PHM) projects.

Methods: The prospective quality improvement projects incorporated three third-year student pharmacists who developed and conducted individual PHM projects over the course of three to seven months. The projects included hypoglycemia screening, hepatitis $C$ virus and human immunodeficiency virus screening, and statin use evaluations for atherosclerotic cardiovascular disease risk reduction. Under the guidance of a clinical pharmacist, students developed project materials, conducted patient chart reviews, and contacted patients to make interventions such as recommendations for therapy, ambulatory patient monitoring, patient education, and arranging provider follow-up. Student impact was evaluated through the number of patients screened, the number of eligible patients contacted, and the total number of interventions or recommendations made. Student time spent was tracked throughout the projects.

Results: Out of 244 patients screened, 198 patients met inclusion criteria and 162 patients were contacted or assessed by a student pharmacist. Students made a total of 319 interventions, including patient education (132), patient monitoring (132),

pharmacotherapy recommendations (28), and arranging follow-up (27). On average students screened 33 patients per month, and, per patient, required 8.6 minutes for eligibility assessment and approximately 6 minutes for telephone interviews.

Conclusion: This report demonstrates that pre-APPE student pharmacists are well-equipped to design and implement PHM projects. Utilization of student pharmacists in similar PHM programs can expand the pharmacist's impact on patient care in the ambulatory care setting.
\end{abstract}

Keywords: Population Health Management, Pharmacy Students, Pharmacy Education, Primary Health Care

\section{Introduction}

How can an organization leverage student pharmacists' skill sets to improve the health of the population served? As population health management (PHM) cements itself as a core component in healthcare, pharmacists need to effectively address this question to improve student pharmacists' education and the health of the population they serve. In essence, PHM is a proactive approach that identifies and executes interventions for patients at high risk of poor health outcomes based on a well-defined clinical parameter. ${ }^{1}$

The United States will experience an estimated shortage of 21,100 to 55,200 primary care physicians by $2032 .^{2}$ This shortage risks worsening healthcare access and quality for patients. Clinical pharmacists, however, are well positioned to mitigate the potential effects of this shortage and improve the triple aim of healthcare developed and defined by the Institute for Healthcare Improvement as (1) improving the experience of care, (2) improving the health of populations, and (3)

Corresponding author: Michael W. Nagy, PharmD Assistant Professor, Clinical Sciences Department Medical College of Wisconsin School of Pharmacy, 8701 W Watertown Plank Rd, Milwaukee, WI 53226, USA Email: mnagy@mcw.edu; Phone: 414-955-2631 reducing the per capita healthcare costs. ${ }^{3}$ When incorporated into primary care teams, clinical pharmacists are a valuable resource for PHM, often addressing each aspect of the triple aim. For example, Altavela et al created a practice model focusing on complex and resource intensive patients, and clinical pharmacist involvement demonstrated a $42 \%$ decrease in 30-day readmission rates and a $20 \%$ decrease in annual medical charges. ${ }^{4}$ Carmichael and colleagues utilized electronic health record (EHR) data within the Veterans Health Administration (VHA) to identify patients failing to meet healthcare effectiveness data and information set (HEDIS) measures. ${ }^{5}$ Data gathered from the EHR was compiled into an interactive "dashboard" which allowed clinical pharmacists to proactively contact applicable patients resulting in over 5,000 additional screenings of patients for hepatitis $\mathrm{C}$ virus (HCV), as well as a $5 \%$ increase in timely hemoglobin A1c measurements in patients at risk for hypoglycemia.

While healthcare systems have identified the value clinical pharmacists bring to PHM, ways to train student pharmacists for an active role in PHM are still under development. ${ }^{6}$ Including PHM as a part of pharmacy training encourages students to become involved in PHM in their professional practice. ${ }^{7}$ Population health management is a recommended component of pharmacy education by the Accreditation Council for Pharmacy Education (ACPE) and the Center for the 
Advancement of Pharmacy Education (CAPE). ${ }^{8,9}$ The American Association of Colleges of Pharmacy established core Entrustable Professional Activities (EPAs) in which new pharmacy graduates should attain competence prior to entering the workforce. ${ }^{10}$ One EPA domain, "population health promoter," includes: identifying patients at risk for prevalent diseases in a population, minimizing adverse drug events and medication errors, and maximizing the appropriate use of medications in a population.

Though coverage of population-based healthcare has expanded in the didactic pharmacy curricula, few reports have described incorporating pharmacy learners in practicing PHM in experiential settings. ${ }^{7}$ Cannon et al incorporated 46 students in advanced pharmacy practice experience (APPE) rotations to assess non-adherence and the need for renal dose adjustments, leading to 3,774 interventions over four years. ${ }^{11}$ In another example, Kaminetzky and colleagues leveraged pharmacy, medicine, and nurse practitioner residents in an interdisciplinary PHM focused training curriculum concentrating on chronic disease state management, which improved PHM skills and increased pharmacy resident confidence in conducting PHM. ${ }^{12}$ Whether woven into an APPE rotation or a year-long post-graduation residency program, practical experience of PHM was mutually beneficial for pharmacy trainees' education and clinical pharmacists' workload capacity. Leveraging the time and energy of student pharmacists to assist in the completion of PHM could enhance the ability of primary care teams to fulfill the triple aim.

This report focused on incorporating third-year student pharmacists in different PHM projects. The primary objective was to evaluate the impact student pharmacists had on the respective clinics by examining the number of patients screened, the number of eligible patients contacted, and the total number of interventions or recommendations made. Student time spent was tracked throughout the projects.

\section{Methods}

The prospective quality improvement projects incorporated three third-year student pharmacists into the workflow for PHM within outpatient clinics of a VHA medical center. Clinical pharmacists within the outpatient clinics were integrated within patient-aligned care teams (PACTs), which included primary care providers (PCP), nurse care managers, social workers, and other specialized healthcare providers. Under the PACT model, clinical pharmacists operated under a scope of practice, which granted them prescriptive authority and laboratory ordering privileges for management of any chronic disease including, but not limited to, diabetes, hypertension, and hyperlipidemia. They were also closely involved in teaching and precepting student pharmacists. Thus, the PACT model offered a unique opportunity for student pharmacists to be directly involved with PHM.
Student PHM projects included a hypoglycemia screening in accordance with the VHAs hypoglycemia safety initiative (HSI), $\mathrm{HCV}$ and human immunodeficiency virus (HIV) screening, and statin use evaluations for atherosclerotic cardiovascular disease risk reduction (ASCVD). ${ }^{13-15}$ Projects were developed by student pharmacists in accordance with the most up-todate clinical guidelines and in collaboration with a clinical pharmacist preceptor. Student pharmacists were employees of the VHA medical center prior to project implementation; however, selection and participation in the quality improvement projects were on a voluntary basis. Students worked independently of one another to create materials for each project, including data collection sheets, phone scripts for speaking with patients, and note templates for documenting patient encounters and recommendations within the EHR. Specific project designs can be found in Table 1. All project materials were reviewed by the clinical pharmacist preceptor with feedback provided for quality improvement.

Student pharmacists were oriented to the EHR system prior to project implementation. Additional training was provided over the course of the data collection period as necessary. Eligible patients for each project were identified utilizing a computerized algorithm and the EHR generated patient dashboards based on pre-selected inclusion and exclusion criteria (Table 1). Each student conducted chart reviews and discussed eligible patients with a pharmacist preceptor. In the $\mathrm{HSI}$ and HCV projects, students contacted patients via telephone and made interventions including recommendations for therapy, ambulatory patient monitoring, patient education, and arranging provider follow-up (Table 1). Specifically, in the HSI project, pharmacotherapy recommendations were made to the patient's PACT pharmacist and patient education was provided on acute hypoglycemia identification and self-management. In contrast, the student pharmacist for the ASCVD project assessed patients for statin eligibility and made recommendations for initiation of statin medication and follow-up to the patient's PCP. A pharmacist preceptor co-signed all student pharmacist notes in the EHR and provided feedback for quality improvement regarding patient interactions and documentation. Descriptive statistics were used to evaluate each project. These projects were not considered as constituting research as defined under 45 CFR 46.102(d).

\section{Results}

A total of 244 patients were identified through the EHR dashboards (Figure 1). Of these, 198 (81\%) patients met inclusion criteria across all projects and $162(66 \%)$ were ultimately contacted or assessed by a student pharmacist. Throughout the project periods, a total of 319 interventions occurred involving multiple PCPs from three outpatient clinics (Table 2).

Of the patients who received direct communication from a student pharmacist $(n=135), 132(97.8 \%)$ received 
ambulatory patient monitoring of new objective data and patient education (Table 2). Monitoring in the HCV project resulted in one positive screening for $\mathrm{HCV}(1 / 110,0.91 \%)$ and no positive screenings for HIV $(0 / 40,0 \%)$. The HSI project intervened on four instances of clinical hypoglycemia $(4 / 25$, $16 \%)$.

Twenty-eight recommendations were made to adjust pharmacotherapy after patient interview or assessment and discussion with a pharmacist preceptor (Table 2). Follow-up was arranged in all three projects for 27 (16.7\%) patients. All patients screening positive for HCV or hypoglycemia had follow-up arranged with a PACT team member.

On average 8.6 minutes were required to assess patients for eligibility as well as prepare for screening prior to patient contact. Approximately 1,500 minutes ( 25 hours) were spent reviewing patient charts over the entire course of all projects. Time spent talking with patients for the HCV and HSI projects totaled 790 minutes with less than 6 minutes spent per patient on average. The average monthly number of patients screened was approximately eight in the HSI project, nine in the ASCVD project, and 16 in the HCV project.

\section{Discussion}

These projects demonstrated the utility of incorporating student pharmacists in PHM through the large number of patients screened, interventions made, and potential increased clinical pharmacist impact. The interventions made by students included engaging patients in screening for infectious diseases, evaluating patient's self-management of care, providing education, and identifying drug related problems with appropriate follow-up. The HCV and HSI projects revealed previously undetected HCV infection and clinical hypoglycemia respectively, while the ASCVD project identified $75 \%$ of patients as eligible for a statin. Students demonstrated the capability of conducting PHM while using critical thinking skills to propose resolutions to clinical problems.

Student pharmacists have the clinical knowledge and skill set needed to address unique disease states in PHM, such as HCV screening, hypoglycemia identification and education, and appropriate statin prescribing. These PHM topics were selected based on complexity to match student pharmacist knowledge and clinical skills to allow for practical application with guided practice. To our knowledge this is the first report incorporating pre-APPE students into PHM during experiential practice, as well as the first report targeting these disease states. A novel aspect of this project was the targeting of different subcategories of the "population health promoter" EPA domain. The HCV project effectively identified at-risk patients for the prevalent disease; the HSI project focused on minimizing hypoglycemic adverse events; the ASCVD project worked toward maximizing statin use within an at-risk population.
When interpreting the number of screenings per month performed by each student pharmacist, it is important to note that students were generally present on site between four and six hours weekly. In addition to the time required for patient workup and telephone interactions, students spent time documenting encounters and discussing clinical aspects of patient care with the clinical pharmacist preceptor. While projects in this report made fewer interventions and saved less pharmacist time compared to the Cannon et al study, the scale of the work was smaller in terms of the number of student pharmacists ( 3 versus 46 students) and duration ( 3 to 7 months versus nearly 4 years). ${ }^{11}$ Also, student pharmacists within the Cannon et al study devoted $50 \%$ to $60 \%$ of their APPE rotation time to PHM. In comparison, this report serves as a model for actively incorporating student pharmacists into PHM prior to full-time clinical rotations.

Incorporation of student pharmacists into PHM offered several advantages to the student, clinic, and patient population. Due to the applicability of PHM to a variety of metrics within chronic disease states, each student took ownership of a project that matched their area of interest. Students gained hands-on experience in project development and management, had the opportunity to exercise written and verbal communication skills, and practiced clinical judgement outside of the classroom. These skillsets are essential in the preparation of students for APPEs and can help to facilitate student adjustment from didactic to practical training. Minimizing this adjustment period enables students to maximize time in their future clinical experiences, empowering them to become competent and capable pharmacists. Student involvement also enabled the pharmacist to expand clinical services. This partnership expanded patient care opportunities at the population level with many possible indirect benefits. For example, the education provided to patients may enhance health literacy, and the act of patient outreach may encourage engagement and improve satisfaction with care.

These projects came with several limitations. Projects were conducted at a single institution where pharmacists have an advanced scope of practice. This model may not reflect the pharmacist's role at other institutions, limiting generalizability to other practice sites. A second limitation was students' varying levels of experience with the EHR prior to project implementation. When incorporating pre-APPE students into projects, resources for onboarding and training can limit feasibility; therefore, leveraging students familiar to the system can decrease the onboarding period.

Future directions include incorporating PHM regularly into student pharmacist introductory pharmacy practice experience (IPPE) rotations. Since project materials have been developed and tested, future students can focus on screening and increase the efficiency and breadth of interventions. Future student PHM projects should incorporate formal student self-evaluations to promote awareness of perceived 
changes in knowledge, skills, and abilities. Survey evaluation could occur at the start of APPE rotations to assess the impact of the student's PHM experience on their ability to transition from didactic to practical training. Finally, measurement of the number of accepted recommendations and long-term clinical outcomes will quantify the impact of student led PHM projects.

\section{Conclusion}

Pharmacy education organizations have identified PHM as an area where new graduates should obtain the knowledge, skills, and abilities needed to successfully perform. This report highlights that pre-APPE student pharmacists can design and implement PHM projects and make significant impacts on the care of ambulatory patients. These projects demonstrate three unique disease states in which clinical pharmacists can engage students to expand clinical pharmacist impact. The novel endeavor can benefit students' education and advance goals of the respective healthcare organization while striving for the triple aim of healthcare.

Acknowledgements: We would like to thank Magdalena Siodlak, Dei Forslund, and Dee McConnell for reviewing and editing the final manuscript.

Conflict of Interest: None

Financial disclosures: None

Support Disclosures: This material is the result of work supported with resources and the use of facilities at the William S. Middleton Memorial Veterans Hospital in Madison, Wisconsin. The contents do not represent the views of the U.S. Department of Veterans Affairs or the United States Government.

Treatment of Human Subjects: IRB determined project was non-HSR

\section{References}

1. Swarthout M, Bishop MA. Population health management: review of concepts and definitions. Am J Health Syst Pharm. 2017;74(18):1405-1411.

2. Association of American Medical Colleges. 2019 Update: the complexities of physician supply and demand: projections from 2017 to 2032. https://aamcblack.global.ssl.fastly.net/production/media/filer_public/3 1/13/3113ee5c-a038-4c16-89af294a69826650/2019_update__the_complexities_of_physician_supply_and_demand__projections_from_2017-2032.pdf. Accessed: May 14, 2019.

3. Berwick DM, Nolan TW, Whittington J. The triple aim: care, health, and cost. Health Aff. 2008;27(3):759-769.

4. Altavela JL, Dorward KM, Sorrento TA, Diehl KM, Wyman CA. Population health management: an independent physician organization approach. Am J Health Syst Pharm. 2017;74(18):1477-1485.
5. Carmichael JM, Meier J, Robinson A, Taylor J, Higgins DT, Patel S. Leveraging electronic medical record data for population health management in the Veterans Health Administration: successes and lessons learned. Am J Health Syst Pharm. 2017;74(18):1447-1459.

6. Knoer SJ, Swarthout MD, Sokn E, et al. The Cleveland Clinic pharmacy population health management summit. Am J Health Syst Pharm. 2018;75(18):1421-1429.

7. Crawford SY. Pharmacists' roles in health promotion and disease prevention. Am J Pharm Educ. 2005;69(4):Article 73.

8. Accreditation Council for Pharmacy Education. Accreditation standards and key elements for the professional program in pharmacy leading to the doctor of pharmacy degree. https://www.acpeaccredit.org/pdf/Standards2016FINAL.pdf. Accessed: April 5, 2019.

9. Medina MS, Plaza CM, Stowe CD, et al. Center for the Advancement of Pharmacy Education 2013 education outcomes. Am J Pharm Educ. 2013;77(8):Article 162.

10. Haines ST, Pittenger AL, Stolte SK, et al. Core entrustable professional activities for new pharmacy graduates. Am J Pharm Educ. 2017;81(1):Article S2.

11. Cannon EC, Zadvorny KB, Sutton SD, et al. Evaluating value of population management activity interventions performed by pharmacy students: a pilot study [published online ahead of print June 13, 2018]. Am J Pharm Educ. https://www.ajpe.org/doi/pdf/10.5688/ajpe6759.

Accessed: April 5, 2019.

12. Kaminetzky CP, Beste LA, Poppe AP, et al. Implementation of a novel population panel management curriculum among interprofessional health care trainees. BMC Med Educ. 2017;17(264):1-8.

13. U.S. Department of Veterans Affairs/U.S. Department of Defense. VA/DoD clinical practice guideline for the management of type 2 diabetes mellitus in primary care. 2017.

https://www.healthquality.va.gov/guidelines/CD/diabetes /VADoDDMCPGFinal508.pdf. Accessed: May 15, 2019.

14. Smith BD, Morgan RL, Beckett GA, et al; Recommendations for the identification of chronic hepatitis $C$ virus infection among persons born during 1945-1965. MMWR Recomm Rep. 2012;61(RR-4):1-32.

15. Stone NJ, Robinson JG, Lichtenstein AH, et al. 2013 $\mathrm{ACC} / \mathrm{AHA}$ guideline on the treatment of blood cholesterol to reduce atherosclerotic cardiovascular risk in adults: a report of the American College of Cardiology/American Heart Association Task Force on Practice Guidelines. Circulation. 2014;129(25 Suppl 2):S1-S45. 
Table 1. Methodology of Student Population Health Management Projects

HCV Project^

7

\begin{abstract}
Duration, months
\end{abstract}
Student Pharmacist

Responsibilities

\section{Simplified Data Collection Process}

Inclusion Criteria

\section{Exclusion Criteria}

- Performed chart reviews

- Contacted patients

- Educated patients on HCV prevalence, risks, and efficacy of current treatments

- Ordered HCV and/or HIV antibody lab screenings

- If unable to reach patients, left voicemails and mailed informational letters on HCV

- Documented work in EHR

- Response to HCV/HIV screening

- Labs: HCV and/or HIV antibody test result(s)

- Informational letters sent

- Telephone messages left

- Terminal illness or life expectancy less than 6 months

- Screened outside the VHA system (determined in EHR)

HCV antibody screen:

- Born from 1945 to 1965 with undocumented HCV status

- Upcoming lab appointment

HIV antibody screen:

- $\quad$ Patients eligible for HCV screening with undocumented HIV status

- Documented terminal illness or life expectancy $<6$ months

- Previously screened outside the VHA system

- Unable to contact

\section{HSI Project^^}

3

- Performed chart reviews

- Contacted patients

- Conducted brief medication history and recorded blood glucose readings

- $\quad$ Educated patients on signs and self-management of hypoglycemia*

- Developed hypoglycemia prevention plan

- Coordinated follow-up

- Documented work in EHR

- Age

- Type of DM

- Labs: SCr and A1c

- DM medications

- Presence of a beta blocker

- $\quad$ SMBG (if available)

- Diagnosis of cognitive impairment or dementia

- Subjective or objective hypoglycemia reported

- Education provided

- Prescribed sulfonylurea or insulin

- Most recent $\mathrm{A} 1 \mathrm{c}<7 \%$

- At least 1 of the following:

- Age $>74$ years

- $\mathrm{SCr}>1.7 \mathrm{mg} / \mathrm{dL}$

- Diagnosed dementia or cognitive impairment

- Admitted to a long term care or inpatient facility

- $\quad$ No A1c or Scr measured within the last 18 months

- Unable to contact
ASCVD Project^^^

3

- Performed chart reviews

- Assessed need for statin therapy

- $\quad$ Formulated statin therapy recommendation

- Coordinated follow-up

- Documented work in EHR

- Age

- Body mass index

- Labs: lipid profile

- 10-year ASCVD risk**

- History of DM or hypertension

- Family history of ASCVD

- Statin use history

- Smoking history

- 40-75 years old

- 10 -year ASCVD risk $\geq 7.5 \%$

- $\quad \mathrm{LDL}>70 \mathrm{mg} / \mathrm{dL}$

- History of ASCVD

- $\mathrm{LDL}<70 \mathrm{mg} / \mathrm{dL}$

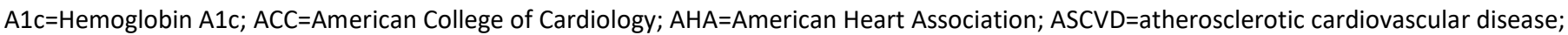
$\mathrm{DM}=$ diabetes mellitus; $\mathrm{EHR}=$ =lectronic health record; $\mathrm{HCV}=$ hepatitis $\mathrm{C}$ virus; $\mathrm{HIV=human} \mathrm{immunodeficiency} \mathrm{virus;} \mathrm{HSI}=$ hypoglycemia safety initiative; LDL=low density lipoproteins; $\mathrm{SCr}=$ serum creatinine; SMBG=self-monitoring of blood glucose; VHA=Veterans Health Administration. *Hypoglycemia is defined as self -monitored blood glucose readings $<70 \mathrm{mg} / \mathrm{dL}$.

**Accessed at http://tools.acc.org/ASCVD-Risk-Estimator-Plus/\#!/calculate/estimate/.

Guidelines utilized for project development:

^ Smith BD, Morgan RL, Beckett GA, et al; Recommendations for the identification of chronic hepatitis $C$ virus infection among persons born during 1945-1965. MMWR Recomm Rep. 2012;61(RR-4):1-32.

$\wedge \wedge$ U.S. Department of Veterans Affairs/U.S. Department of Defense. VA/DoD clinical practice guideline for the management of type 2 diabetes mellitus in primary care. 2017:1-160. https://www.healthquality.va.gov/guidelines/CD/diabetes/VADoDDMCPGFinal508.pdf. Accessed May 15, 2019.

^^^Stone NJ, Robinson JG, Lichtenstein AH, et al. 2013 ACC/AHA guideline on the treatment of blood cholesterol to reduce atherosclerotic cardiovascular risk in adults: a report of the American College of Cardiology/American Heart Association Task Force on Practice Guidelines. Circulation. 2014;129(25 Suppl 2):S1-S45. 
Table 2. Interventions Provided by Student Pharmacists

\begin{tabular}{|c|c|c|c|c|}
\hline \\
\hline & $\begin{array}{c}\text { HCV } \\
(n=110)\end{array}$ & $\begin{array}{c}\text { HSI } \\
(n=25)\end{array}$ & $\begin{array}{l}\text { ASCVD } \\
(n=27)\end{array}$ & Total \\
\hline Number of Total Interventions, $\mathrm{n}$ & 218 & 61 & 40 & 319 \\
\hline Education, n (\%) & $110(100)$ & $22(88)$ & N/A & $132(97.8)$ \\
\hline Pharmacotherapy Recommendation, $\mathrm{n}(\%)^{*}$ & $0(0)$ & $8(32)$ & $20(74.1)$ & $28(17.2)$ \\
\hline Ambulatory Patient Monitoring, $\mathrm{n}(\%)$ & $107(97.3)$ & $25(100)$ & N/A & $132(97.8)$ \\
\hline Follow-up arranged, $\mathrm{n}(\%)$ & $1(0.9)$ & $6(24)$ & $20(74.1)$ & $27(16.7)$ \\
\hline Patients assessed per month, mean & 16 & 8 & 9 & 33 \\
\hline \multicolumn{5}{|l|}{ Time committed per patient in minutes } \\
\hline Preparation, mean & 5 & 15 & 22 & 8.6 \\
\hline Screening, mean & 5 & 9.6 & N/A & 5.7 \\
\hline
\end{tabular}

ASCVD=atherosclerotic cardiovascular disease; $\mathrm{HCV}=$ hepatitis $\mathrm{C}$ virus; $\mathrm{HSI}=$ hypoglycemia safety initiative Note: percent for individual projects reflect total patients included in that project only

Figure 1. Consort Diagram for Patient Inclusion and Exclusion

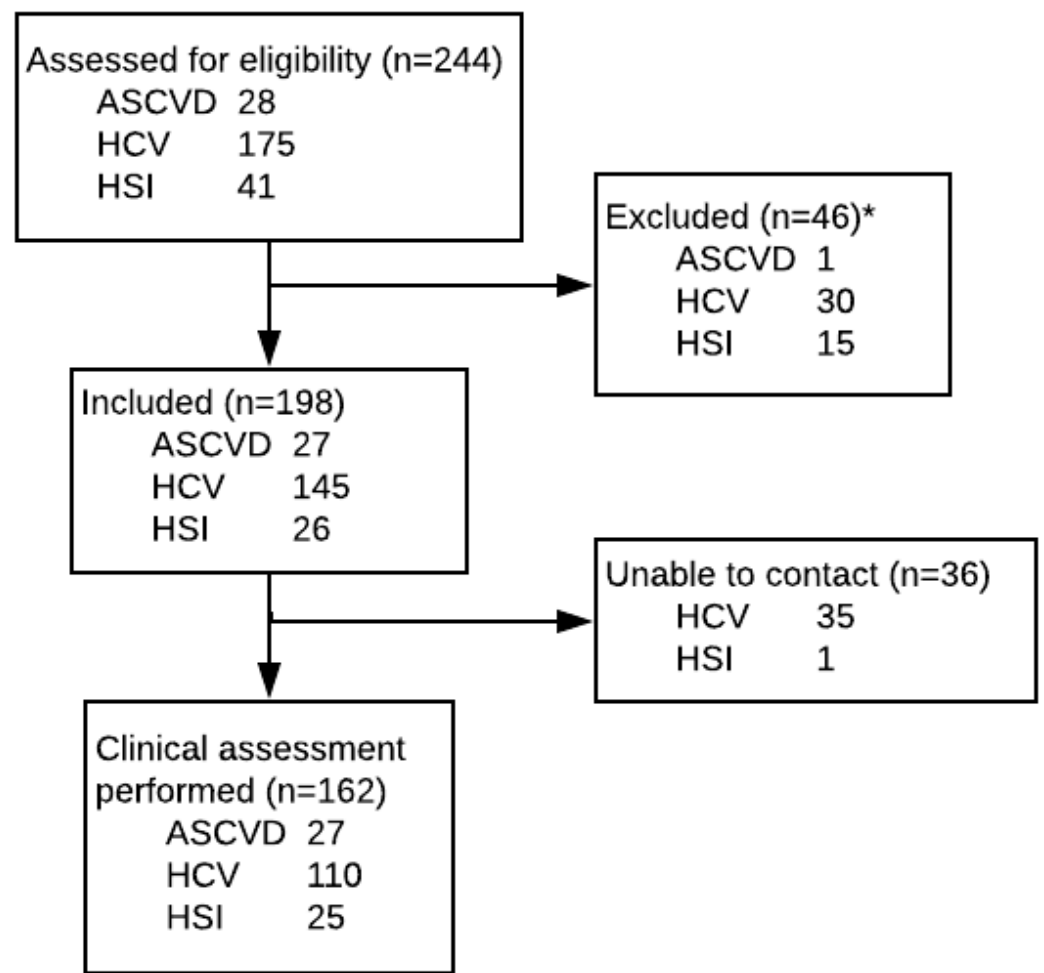

*Excluded for not meeting inclusion criteria of respective projects in Table 1

ASCVD=atherosclerotic cardiovascular disease; $\mathrm{HCV}=$ hepatitis $\mathrm{C}$ virus; $\mathrm{HSI}=$ hypoglycemia safety initiative 